\title{
Instrumentos de Avaliação em Espondilite Anquilosante
}

\section{Outcome Measures in Ankylosing Spondylitis}

\author{
Themis Mizerkowski Torres ${ }^{(1)}$, Rozana Mesquita Ciconelli(2)
}

\section{RESUMO}

A espondilite anquilosante (EA) é uma doença inflamatória crônica caracterizada por acometimento predominante do esqueleto axial. Ocorre de forma insidiosa e é potencialmente debilitante, levando à redução na qualidade de vida dos indivíduos acometidos. A sua etiopatogenia ainda não está totalmente esclarecida, dificultando estratégias no seu diagnóstico e manejo. O avanço da terapia com agentes biológicos veio reforçar discussões sobre a melhor forma de avaliação destes pacientes. Nesta revisão, discutimos os principais instrumentos utilizados para avaliar pacientes com EA e o consenso do grupo internacional (ASAS working group Assessments in Ankylosing Spondylitis Working Group) determinado no OMERACT IV (Outcome Measures in Rheumatology).

Palavras-chave: espondilite anquilosante, instrumentos, qualidade de vida.

\section{INTRODUÇÃO}

A Espondilite Anquilosante (EA) é uma doença sistêmica inflamatória crônica caracterizada por acometimento primário do esqueleto axial com prevalência de $0,9 \%$ na população geral $^{(1)}$. Tem a característica de acometer predominantemente indivíduos do sexo masculino na segunda e terceira décadas de vida, gerando um forte impacto socioeconômico e na qualidade de vida dos pacientes ${ }^{(2,3)}$. Recentemente, tem sido demonstrado que drogas anti-TNF são potencialmente modificadoras da atividade da doença, atuando na melhora da capacidade funcional, mobilidade da coluna e nas articulações periféricas e enteses ${ }^{(4-6)}$. Desta maneira, há a necessidade de se definir medidas de avaliação específicas para o acompanhamento e tratamento de pacientes com EA.

Definir atividade de doença em afecções crônicas é uma tarefa difícil. A dificuldade aumenta quando se depara com doenças reumatológicas de evolução lenta e ausência de um marcador laboratorial definido, como a EA. Elabora-se, então, diversos instrumentos para a avaliação da atividade, a maioria em forma de questionários.

\section{ABSTRACT}

Ankylosing spondylitis is a chronic and progressive disease involving predominantly the axial skeleton. It is insidious and potentially debilitating, compromising the quality of life of patients suffering from the disease. The etiopathogenesis is still uncertain, which difficult strategies in its diagnosis and treatment. Advances in biological therapies are reforcing discussions in the best way of managing the disease. In this paper, we revise the outcome instruments available for ankylosing spondylitis and the consensus from the ASAS working group (Assessments in Ankylosing Spondylitis Working Group) established at the OMERACT IV (Outcome Measures in Rheumatology).

Keywords: ankylosing spondylitis, instruments, quality of life.

A abordagem pode ser feita através de modelos genéricos ou específicos. Os questionários genéricos refletem o impacto da doença sobre a vida do indivíduo numa larga variedade de populações e envolvem domínios como atividade funcional, incapacidade, estresse físico e emocional. Os específicos oferecem informações mais especializadas sobre uma área de interesse primário, podendo ser uma doença, população (ex: crianças) ou funções como sono ou atividade sexual, utilizados quando se necessita de informações mais detalhadas em relação a aspectos da própria doença ${ }^{(7)}$.

Existem vários instrumentos de avaliação em EA, sendo os principais os descritos abaixo:

\section{ESPECÍFICOS}

- BASDAI (Bath Ankylosing Spondylitis Disease Activity Index $)^{(8)}$ : questionário desenvolvido para medir a atividade da doença. Provou ser válido, reprodutível e sensível a mudanças. Consiste em seis questões que abordam domínios relacionados à fadiga, dor na coluna, dor e sintomas

\footnotetext{
Disciplina de Reumatologia da Universidade Federal de São Paulo (UNIFESP). Recebido em 14/07/05. Aprovado, após revisão, em 28/01/06.

1. Pós-graduanda da Disciplina de Reumatologia da UNIFESP.

2. Médica Assistente da Disciplina de Reumatologia da UNIFESP.

Endereço para correspondência: Themis Mizerkowski Torres, Rua Azevedo Macedo, 57/44, Vila Mariana, São Paulo, SP, Brasil, e-mail: themistorres@uol.com.br
} 
articulares, dor devido ao acometimento das enteses, e duas questões relacionadas à qualidade e quantidade de rigidez matinal. O escore é medido em escala visual analógica $(\mathrm{EVA})$ de 0 a $10(0=$ bom; 10 = ruim $)$. É considerado, atualmente, um dos mais importantes instrumentos para a utilização em ensaios clínicos.

- BASFI (Bath Ankylosing Spondylitis Functional In$d e x)^{(9)}$ : medida de avaliação da capacidade funcional. Inclui oito itens relacionados a atividades da vida diária e dois itens que medem as habilidades do paciente em lidar com o seu dia-a-dia. $\mathrm{O}$ escore é de 0 a 10 em EVA $(0=$ bom; $10=$ ruim). Constitui, junto com o Dougados' Functional Index (DFI), medida escolhida para o item função dos domínios definidos pelo grupo ASAS ${ }^{(10,11)}$.

- BASRI (Bath Ankylosing Spondylitis Radiology Index $)^{(12)}$ : método de graduação radiográfica do acometimento da coluna, em uma escala de 0 a $4(0=$ normal, $1=$ lesões suspeitas, 2 = quadratura vertebral com erosões e esclerose, 3 = lesões mais difusas com formação de sindesmófitos, 4 $=$ anquilose $)$. As localizações escolhidas para a abordagem são bacia, coluna cervical e lombar, resultando em um escore de 2 a 12.

- BAS-G (Bath Ankylosing Spondylitis Global Score ${ }^{(13)}$ : mede a avaliação global do paciente. São duas questões em EVA de 0 a $10(0=$ bom; $10=$ ruim $)$ que abordam o efeito da doença na percepção global do paciente, na última semana e nos últimos seis meses.

- BASMI (Bath Ankylosing Spondylitis Metrology Index $)^{(14)}$ : engloba medidas da coluna no intuito de definir o status axial do paciente. As medidas envolvidas são: rotação cervical, distância occipito-parede, flexão lombar, teste de Schober modificado e distância intermaleolar. Cada medida é convertida em um escore de 0 a 10 ( $0=$ bom; $10=$ ruim $)$.

- ASQoL (Ankylosing Spondylitis Quality of Life Questionnaire)(15): instrumento de medida de qualidade de vida (QoL). Engloba 18 questões com resposta sim ou não, resultando em um escore de 0 a 18 , com o maior valor implicando em uma pior qualidade de vida. É o único desenvolvido originalmente para a medida específica de qualidade de vida em EA.

- HAQ-S (Health Assessment Questionnaire Spondylitis ${ }^{(16)}$ : versão do HAQ para EA, mede função. Difere do HAQ original por apresentar mais cinco questões relacionadas a atividades envolvendo a coluna, como dirigir com o carro em ré ou carregar sacolas pesadas. A escala varia de 0 (sem dificuldade) a 3 (incapacidade).

- AS-AIMS2 (Arthritis Impact Measurement Scales 2 - Ankylosing Spondylitis) ${ }^{(17)}$ : versão do AIMS-2 para a
EA, mede qualidade de vida. Engloba uma nova dimensão denominada mobilidade espinhal, composta de seis ou quatro itens, dependendo da habilidade do paciente em dirigir automóveis. O escore é calculado de 0 (saúde perfeita) a 10.

- DFI (Dougados' Functional Index) ${ }^{(18)}$ : instrumento para a avaliação da capacidade funcional. Contém 20 itens relacionados à capacidade do paciente em realizar tarefas da vida diária. Todas a questões iniciam-se por "Você consegue", com três modalidades de resposta: 0 (sim, sem dificuldade), 1 (sim, com dificuldade) e 2 (não). O escore é calculado como a soma de todas as respostas (0-40).

- PGI ( Patient Generated Index ${ }^{(19)}$ : medida individual de qualidade de vida. Já foi validado para o uso em lombalgia e menorragia, dermatite e apnéia do sono. O cálculo do escore envolve três estágios: no primeiro, o paciente faz uma lista de cinco áreas da sua vida mais afetadas pela doença. No segundo, escolhe, em uma escala de 0 (pior) a 10 (melhor), como se comporta a sua vida em cada área determinada. No terceiro, faz uma escala de prioridades para a melhora atribuindo pontos de 0 a 14 . O escore é calculado multiplicando-se o ponto dado no segundo estágio à proporção encontrada no terceiro estágio. A somatória dos escores para cada área resulta em um escore final de 0 a 10 (melhor qualidade de vida).

- RLDQ (Revised Leeds Disability Questionnaire) ${ }^{(20)}$ : medida relacionada à capacidade funcional. Contém 16 itens distribuídos em 4 domínios: mobilidade, inclinação, movimentos cervicais e postura. $\mathrm{O}$ escore situa-se entre 0 (sem dificuldade) e 3 (incapacidade) para cada item, sendo o escore final variando de 0 a 48 , com os maiores valores indicando maior incapacidade.

- Body Chart $^{(20)}$ : avalia dor global. O método consiste em pedir ao paciente para escolher, em um manequim, as áreas do corpo mais doloridas. Para cada uma, é dada um nota de 0 (dor leve) a 4 (dor intensa). Realiza-se a soma das notas, resultando no escore final, sem haver valor máximo.

- SASSS (Stoke Ankylosing Spondylitis Spine Score)(21): medida de avaliação radiológica. As localizações escolhidas para a graduação são as articulações sacroilíacas $(0=$ normal, $1=$ borramento, $2=1+$ esclerose ou pseudoalargamento, $3=$ $2+$ erosões ou pontes ósseas, 4 = anquilose) e coluna lombar ( 1 = erosão, esclerose ou quadratura, 2 = sindesmófitos, 3 $=$ anquilose $)$. A avaliação da coluna é feita tanto posterior quanto anteriormente, englobando o bordo inferior de T12 ao bordo superior de S1. O máximo do escore composto é de 72. Atualmente, este método foi modificado, surgindo o M-SASSS (modified Stoke Ankylosing Spondylitis Spine 
Score $)^{(22)}$, diferindo do original por apenas abordar a parte anterior da coluna lombar e por incluir a análise da coluna cervical (do bordo inferior de C2 ao bordo superior de T1). Em estudo recente, comparando os três métodos para avaliação radiológica da EA (BASRI, SASSS e M-SASSS), o M-SASSS demonstrou ser o melhor método para a análise da progressão radiológica na espondilite anquilosante. ${ }^{(23)}$

\section{GENÉRICOS}

- SF-36 (Medical Outcomes Study (MOS) 36-Item Short Form): instrumento mundialmente empregado em estudos envolvendo qualidade de vida. Apresenta oito domínios: capacidade funcional, aspectos físicos, aspecto emocional, saúde mental, aspectos sociais, vitalidade, dor e percepção geral de saúde. O escore é de 0-100, com valores maiores indicando melhor qualidade de vida. Já está traduzido e validado para o uso no nosso país ${ }^{(24)}$.

- EUROQoL: inclui valores do estado de saúde e tem grande potencial para aplicação em avaliações econômicas. Apresenta duas seções: EQ-5D e EQ-VAS. A primeira (EQ-5D) contém cinco itens cobrindo os domínios de mobilidade, cuidados próprios, atividade habitual, dor/desconforto e ansiedade/depressão. Cada item é graduado em uma escala de 1 (sem problemas) a 3 (incapacidade/ problemas graves). Os escores situam-se entre $-0,59$ a 1,00 , onde 1,00 é considerado saúde perfeita e escores menores que 0 são definidos como piores que a morte. A segunda seção (EQ-VAS) inclui uma EVA em que o paciente gradua seu estado geral de saúde de 0 (pior imaginável) a 100 (melhor imaginável) ${ }^{(25)}$.

- WHODAS II (World Health Organisation Disability Assessment Schedule II): questionário multidimensional que pode ser utilizado para medir o nível de incapacidade. Inclui seis domínios (compreensão e comunicação, relacionamentos, participação na sociedade, atividades relacionadas ao trabalho e serviços de casa, cuidados próprios, mobilidade). É constituído por 36 questões e o escore final é de 0 (melhor) a 100 (pior) ${ }^{(25,26)}$.

Dentre todos estes instrumentos, houve a necessidade de se definir a melhor forma de avaliação. Desta maneira, em 1995, foi formado um grupo internacional (ASAS) que estabeleceu as áreas principais de enfoque na abordagem aos pacientes com EA. ${ }^{(10,11,27,28)}$

\section{ASAS WORKING GROUP - CONSENSO}

O ASAS Working Group é um grupo formado por médicos, pesquisadores, pacientes e representantes de indústrias farmacêuticas, com experiência em EA, englobando mais de 20 países. Baseado em uma combinação de consensos especializados e análises estatísticas, estabeleceu os principais domínios recomendados para a avaliação de pacientes com EA. Estes domínios estão distribuídos em três áreas principais (core sets), conforme Tabela 1 :

- tratamento com medicações que controlam a doença (DC-ART).

- tratamento com medicações que controlam os sintomas (SM - ARD)/ fisioterapia.

- avaliação clínica.

Para cada domínio, foram então escolhidos um ou mais instrumentos específicos dentre 105 identificados na literatura. Trinta e cinco destes foram eliminados por serem considerados irrelevantes ou inviáveis e os restantes foram analisados segundo critérios de simplicidade, confiabilidade, relevância clínica e capacidade de discriminação. $\mathrm{O}$ resultado da seleção final é mostrado na Tabela 2 .

TABELA 1

Domínios Selecionados para Estudos Envolvendo DC-ART, SM-ARD / Fisioterapia, Avaliação Clínica

\begin{tabular}{|c|c|c|}
\hline DC-ART & SM-ARD/FISIOTERAPIA & AVALIAÇÃO CLÍNICA \\
\hline função & função & função \\
\hline dor & dor & dor \\
\hline mobilidade da coluna & mobilidade da coluna & mobilidade da coluna \\
\hline rigidez da coluna & rigidez da coluna & rigidez da coluna \\
\hline $\begin{array}{l}\text { avaliação global do } \\
\text { paciente }\end{array}$ & $\begin{array}{l}\text { avaliação global do } \\
\text { paciente }\end{array}$ & $\begin{array}{l}\text { avaliação global do } \\
\text { paciente }\end{array}$ \\
\hline $\begin{array}{c}\text { articulações periféricas/ } \\
\text { enteses }\end{array}$ & & $\begin{array}{c}\text { articulações } \\
\text { periféricas/enteses }\end{array}$ \\
\hline $\begin{array}{l}\text { provas de atividade } \\
\text { inflamatória }\end{array}$ & & $\begin{array}{l}\text { provas de atividade } \\
\text { inflamatória }\end{array}$ \\
\hline \multicolumn{3}{|l|}{ radiografia da coluna } \\
\hline \multicolumn{3}{|l|}{ radiografia do quadril } \\
\hline fadiga & & \\
\hline
\end{tabular}

*DC-ART - tratamento com medicações que controlam a doença. SM-ARD - tratamento com medicações que controlam os sintomas.

\section{FUNÇÃO}

O domínio função relaciona-se à atividade da doença e danos. O BASFI e DFI estão entre os instrumentos escolhidos para a sua avaliação, ambos demonstrando propriedades válidas e confiáveis. Há, no entanto, diferenças entre eles, descritas em estudo feito por Ruof e Stucki(29) (Tabela 3):

Atualmente, O BASFI é a medida de avaliação funcional mais utilizada nos estudos clínicos. 
TABELA 2

INSTRUMENTOS ESPECÍ́FICOS PARA CADA DOMÍNIO RELACIONADO A DROGAS CONTROLADORAS DA DOENÇA (DC-ART), DROGAS MODIFICADORAS DE SINTOMAS (SM-ARD), FISIOTERAPIA, AVALIAÇÃO CLÍNICA

\begin{tabular}{|c|c|}
\hline DOMÍNIO & INSTRUMENTOS \\
\hline Função & BASFI OU DFI \\
\hline Dor & $\begin{array}{c}\text { EVA, última semana, devido à coluna à } \\
\text { noite e EVA, última semana, } \\
\text { devido à coluna }\end{array}$ \\
\hline Mobilidade da coluna & $\begin{array}{l}\text { Expansibilidade torácica e Schober } \\
\text { modificado e distância } \\
\text { occipito-parede }\end{array}$ \\
\hline Avaliação global do paciente & EVA, última semana \\
\hline Rigidez & $\begin{array}{c}\text { Duração da rigidez matinal, coluna, } \\
\text { última semana }\end{array}$ \\
\hline $\begin{array}{c}\text { Articulações periféricas e } \\
\text { enteses }\end{array}$ & $\begin{array}{c}\text { Número de articulações inflamadas } \\
\text { (44 articulações); não há preferência } \\
\text { para instrumento utilizado para a } \\
\text { avaliação de enteses }\end{array}$ \\
\hline $\begin{array}{l}\text { Provas de atividade } \\
\text { inflamatória }\end{array}$ & VHS \\
\hline Radiografias da coluna & $\begin{array}{c}\text { Incidências ântero-posterior e lateral } \\
\text { da coluna lombar e cervical } \\
\text { e pelve (sacroilíacas e quadris) }\end{array}$ \\
\hline Radiografias dos quadris & ver radiografias da coluna \\
\hline Fadiga & $\begin{array}{c}\text { não há preferência por instrumento } \\
\text { específico }\end{array}$ \\
\hline
\end{tabular}

*BASFI - Bath Ankylosing Functional Index; DFI - Dougados Functional Index; EVA - escala visual analógica; VHS - velocidade de hemossedimentação.

\section{DOR}

Recomenda-se a avaliação da dor através de dois instrumentos:

-EVA 0-100 mm para dor noturna.

-EVA 0-100 mm para dor, sem predominância de horário.

Ambos os instrumentos estão relacionados à dor na coluna devido à EA, na última semana.

Vários estudos utilizam o BASDAI, que contém três medidas de EVA para a avaliação de dor e desconforto:

- dor no pescoço, coluna ou quadril.

- dor/edema em outras articulações.

- desconforto em áreas sensíveis ao tato ou à pressão.

Os dois últimos itens relacionam-se a articulações periféricas. Esta avaliação é feita em outro domínio pelo consenso do ASAS working group.

\section{MOBILIDADE DA COLUNA}

A expansibilidade torácica, o teste de Schober modificado e a distância occipito-parede estão entre as medidas selecionadas para representar este domínio. A versão do teste de Schober (Tabela 4) foi padronizada tendo em vista as várias modificações realizadas na sua execução.

\section{RIGIDEZ DA COLUNA}

Avaliada pela duração da rigidez matinal na última semana. Há dois componentes do BASDAI relacionados a este item:

- EVA indicando a intensidade de rigidez matinal na última semana.

- duração da rigidez matinal na última semana.

TABELA 3

DifERENÇAS E LimitaÇÕes DO BASFI E DFI

\begin{tabular}{|c|c|c|}
\hline BASFI & DFI & LIMITAÇÕES \\
\hline 10 itens & 20 itens & DFI demanda mais tempo para ser completado \\
\hline escore baseado em $10 \mathrm{~cm}$ EVA & $\begin{array}{l}\text { escore baseado } \\
\text { em escala de } 3 \text { pontos }\end{array}$ & $\begin{array}{c}\text { escala do DFI mais limitada em relação à expressão } \\
\text { de mudanças }\end{array}$ \\
\hline $\begin{array}{l}\quad 7 \text { itens similares ao DFI; } \\
3 \text { itens adicionais que melhoram a validade de } \\
\text { conteúdo e o espectro de dificuldade dos itens }\end{array}$ & $\begin{array}{l}7 \text { itens similares ao BASFI; } \\
\text { alguns itens redundantes e outros r } \\
\text { elacionados a sintomas e não à função }\end{array}$ & $\begin{array}{l}\text { a validade de conteúdo e de construto do DFI } \\
\text { inferior à do BASFI }\end{array}$ \\
\hline $\begin{array}{l}\text { capaz de discriminar o efeito de } \\
3 \text { semanas de tratamento com fisioterapia }\end{array}$ & $\begin{array}{l}\text { não é capaz de discriminar o efeito de } \\
3 \text { semanas de tratamento com fisioterapia }\end{array}$ & $\begin{array}{c}\text { a distribuição dos escores do DFI mostram tendência } \\
\text { a escores normais, impedindo a percepção de melhora } \\
\text { em pacientes com incapacidade leve nos estudos } \\
\text { envolvendo fisioterapia }\end{array}$ \\
\hline
\end{tabular}

*BASFI - Bath Ankylosing Functional Index; DFI - Dougados Functional Index. 
TABELA 4

Teste de Schober Modificado

1) Com o paciente de pé, fazer uma marca na região lombar, no meio de uma linha imaginária unindo as duas espinhas ilíacas póstero-superiores

2) Fazer uma nova marca $10 \mathrm{~cm}$ acima da primeira

3) Pedir para o paciente fletir o tronco ao máximo, mantendo os joelhos estendidos

4) Medir a distância entre as duas marcas

5) A distância normal deve ultrapassar $15 \mathrm{~cm}$

A medida de intensidade em uma escala de 0-100 mm aliada à duração tem demonstrado ser um melhor parâmetro para a abordagem da rigidez quando comparada à duração apenas.

\section{AVALIAÇÃO GLOBAL DO PACIENTE}

É o estado geral de saúde e a escolha da sua medida é por EVA na última semana.

Difere do BAS-G, pois este inclui uma escala que mede o estado de saúde nos últimos seis meses.

\section{ARTICULAÇÕES PERIFÉRICAS/ENTESES}

A abordagem das articulações periféricas é feita pela contagem de articulações inflamadas. As 44 articulações incluídas na contagem são: esternoclaviculares, acromioclaviculares, ombros, cotovelos, punhos, joelhos, tornozelos, 10 metacarpofalangianas, 10 interfalangianas proximais das mãos, 10 metatarsofalangianas.

Em relação às enteses, não há instrumento recomendado no momento. $\mathrm{O}$ único instrumento validado na literatura é o Mander Enthesitis Index (MEI) ${ }^{(30)}$, considerado inviável para a inclusão no consenso. Um novo instrumento criado recentemente, o Maastricht Ankylosing Spondylitis Enthesitis Score $(\text { MASES })^{(31)}$, tem demonstrado ser válido e viável, porém ainda necessitando verificar a sua sensibilidade a mudanças. Uma outra forma de avaliar as enteses é aplicar uma das questões do BASDAI relacionada à dor à pressão ou ao tato.

\section{PROVAS DE ATIVIDADE INFLAMATÓRIA}

O parâmetro laboratorial definido no consenso é a velocidade de hemossedimentação (VHS), pelo baixo custo e facilidade de execução. Estudos recentes demonstram que marcadores de fase aguda estão geralmente em níveis baixos na EA, correlacionando-se pouco com a gravidade da doença ${ }^{(32)}$. Porém, algumas avaliações sugerem que estes marcadores podem estar relacionados à responsividade no DC-ART, refletindo em novas informações sobre os efeitos da terapia biológica.

\section{RADIOGRAFIAS DA COLUNA E QUADRIS}

Os locais escolhidos para a avaliação radiográfica são: coluna cervical, coluna lombar e pelve (sacroilíacas e quadris). Porém, não está definido como acessar a coluna torácica ou como deve ser feito o acompanhamento. Além disso, ainda não estão estabelecidas quais alterações realmente expressam atividade da doença, importante questão para a escolha de um método ideal de escore.

Os instrumentos mais utilizados nos estudos clínicos são o Stoke Ankylosing Spondylitis Spine Score (SASSS) e o Bath Ankylosing Spondylitis Radiology Index (BASRI). Ambos apresentam boa reprodutibilidade e validade, porém a capacidade de detectar progressão da doença em l ou 2 anos é baixa ${ }^{(23)}$. Recentemente, houve a publicação de um novo método de avaliação utilizando a ressonância magnética (RM) da coluna. Este método foi testado em 20 pacientes, sendo efetivo na doença ativa. Necessita, porém, de estudos com um maior número de pacientes para avaliar os escores de atividade e cronicidade. ${ }^{(33)}$

\section{FADIGA}

Nenhum instrumento específico foi selecionado para a avaliação de fadiga, por não haver nenhum relevante na literatura. Estudos recentes têm demonstrado que os itens relacionados à fadiga do BASDAI, SF-36 e índice de fadiga multifatorial são boas alternativas para a avaliação. ${ }^{(34)}$

\section{MELHORA DO TRATAMENTO COM DROGAS MODIFICADORAS DOS SINTOMAS E CONTROLADORAS DE DOENÇA}

O grupo ASAS escolheu quatro domínios para a definição de melhora no tratamento com drogas modificadoras de sintomas $^{(35)}$ : função (BASFI), dor (EVA), avaliação global do paciente (EVA), inflamação (média da rigidez matinal no escore do BASDAI ou rigidez matinal de, no máximo, 120 minutos).

Todos os domínios estão em uma escala de 0-100. A melhora (ASAS 20) é definida como sendo maior ou igual a $20 \%$ ou maior ou igual a 10 unidades, em uma escala de 0 a 100 em cada um de três domínios, sem haver piora maior ou igual a $20 \%$ ou maior ou igual a 10 unidades no quarto domínio. O contrário vale como critério de piora. A remissão parcial é definida como nível baixo de atividade de doença, o que significa menos de 20 unidades na escala de 0-100 em cada um dos quatro domínios. 
Para o tratamento com drogas anti-TNF, o grupo ASAS elaborou um consenso descrito abaixo ${ }^{(36)}$ :

\section{CONCLUSÃO}

Com o advento dos agentes biológicos e os seus surpreendentes efeitos em doenças reumáticas como a EA, os esforços para o desenvolvimento de medidas de avaliação estão em evidente crescimento. O grupo ASAS selecionou parâmetros ideais para a abordagem de pacientes com EA, como a avaliação da função, dor, rigidez, fadiga, mobilidade da coluna, articulações periféricas, avaliação global do paciente e exames de atividade inflamatória e radiográficos. Pela importância da qualidade de vida como medida de desfecho em estudos clínicos, questionários como o SF-36 e ASQoL têm sido cada vez mais utilizados e talvez devessem ter seu lugar nos parâmetros de avaliação definidos internacionalmente.

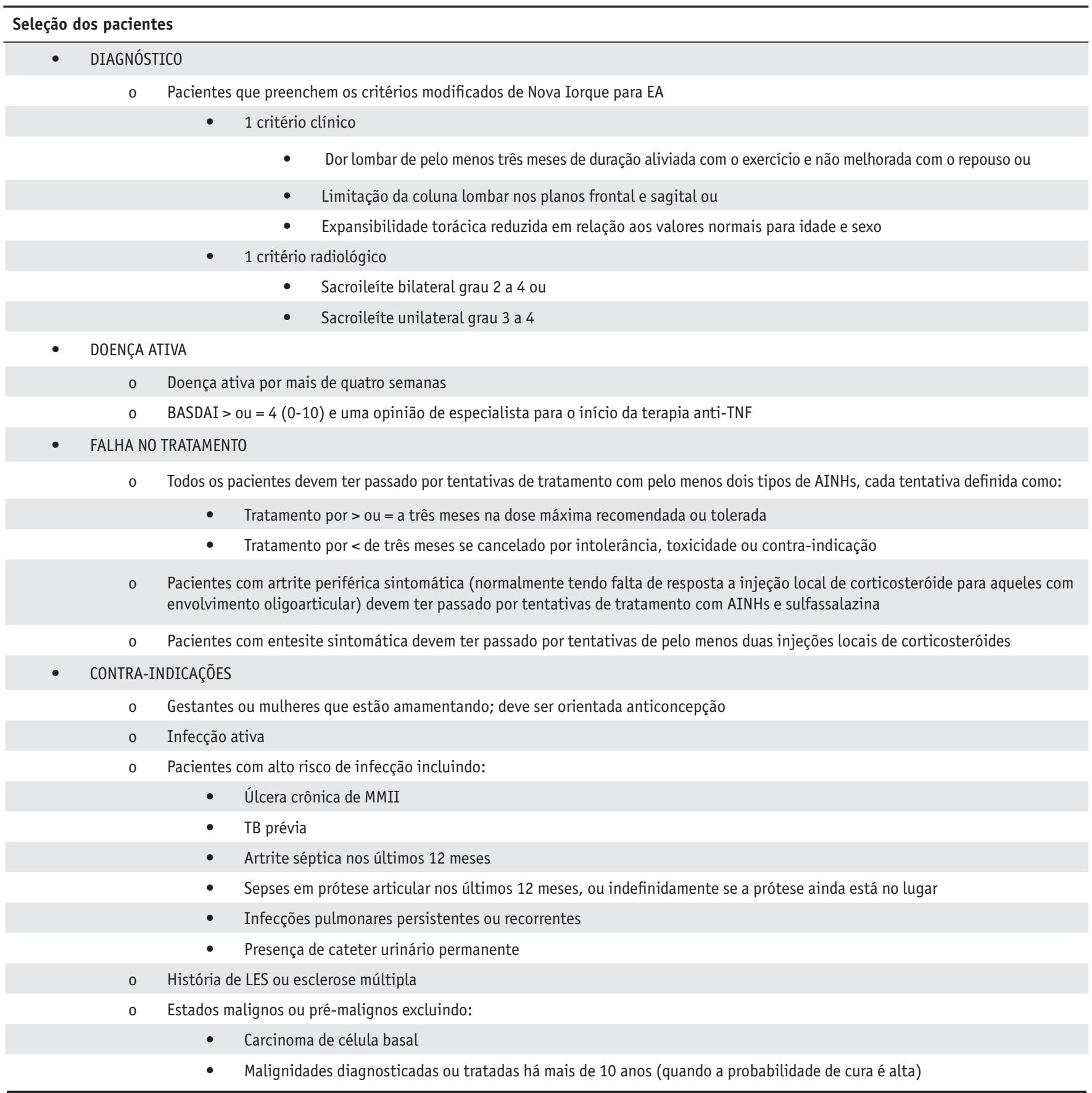




\begin{tabular}{|c|c|c|}
\hline \multicolumn{3}{|c|}{ Avaliação da doença } \\
\hline \multicolumn{3}{|c|}{ - $\quad$ CORE SET ASAS } \\
\hline & 0 & Função (BASFI ou DFI) \\
\hline & 0 & $\begin{array}{l}\text { Dor (EVA, na última semana e da coluna durante a noite devido à EA e EVA, na última semana e } \\
\text { da coluna devido à EA) }\end{array}$ \\
\hline & 0 & $\begin{array}{l}\text { Mobilidade da coluna (expansão torácica e Teste de Schober modificado e distância occipito-parede e } \\
\text { flexão lombar lateral) }\end{array}$ \\
\hline & 0 & Avaliação global do paciente (EVA, última semana) \\
\hline & 0 & Rigidez (duração da rigidez matinal da coluna na última semana) \\
\hline & 0 & $\begin{array}{l}\text { Articulações periféricas e enteses (número de articulações edemaciadas ( } 44 \text { articulações), escore para entesites como os } \\
\text { desenvolvidos em Maastrich, berlim ou São Francisco }\end{array}$ \\
\hline & 0 & Provas de atividade inflamatória (PCR ou VHS) \\
\hline & 0 & Fadiga (EVA) \\
\hline \multicolumn{3}{|c|}{ - $\quad$ BASDAI } \\
\hline & 0 & EVA - fadiga na última semana \\
\hline & 0 & EVA - dor cervical, lombar, ou em quadris na última semana \\
\hline & 0 & EVA - dor/ inflamação em outras articulações que não região cervical, coluna lombar ou quadris na última semana \\
\hline & 0 & EVA - desconforto em quaisquer áreas sensíveis ao tato ou à pressão na última semana \\
\hline & 0 & EVA - rigidez matinal na última semana \\
\hline & 0 & Duração e intensidade (EVA) - rigidez matinal (mais de 120 min) \\
\hline
\end{tabular}

\section{Avaliação da resposta}

- $\quad$ CRITÉRIO DE RESPOSTA

- BASDAI: $50 \%$ de mudança relativa ou mudança absoluta de 2 (escala de 0-10) e opinião de especialista: Continuação sim/não

- $\quad$ TEMPO DE AVALIAÇÃO

o $\quad$ Entre 6 e 12 semanas

\section{REFERÊNCIAS}

1. Braun J, Bollow M, Remlinger G, Eggens U, Rudwaleit M, Distler A et al: Prevalence of spondylarthropathies in HLA-B27 positive and negative blood donors. Arthritis Rheum 41: 58-67, 1998.

2. Braun J, Sieper J: Inception cohorts for spondyloarthropathies. Z Rheumatol 59:11, 2000.

3. Zink A, Braun J, Listing J, Wollenhaupt J: Disability and handicap in rheumatoid arthritis and ankylosing spondylitis - results from the German rheumatological database. J Rheumatol 27: 613-22, 2000 .

4. Braun J, Brandt J, Listing J, Zink A, Alten R, Krause A et al. Treatment of active ankylosing spondylitis with infliximab-a double-blind, placebo-controlled multicenter trial. Lancet 359:1187-93, 2002
5. Gorman JD, Sack KE, Davis JC Jr: Treatment of ankylosing spondylitis by inhibition of tumor necrosis factor $\alpha$. N Engl J Med 346: 1349-56, 2002.

6. Barkham N, Kong KO, Tennant A et al: The unmet need for anti-tumour necrosis factor (anti-TNF) therapy in ankylosing spondylitis. Rheumatology (Oxford) 44 : 1277-81, 2005.

7. Ciconelli RM. Medidas de avaliação de qualidade de vida: Rev Bras Reum 43: IX - XIII, 2003.

8. Calin A, Nakache JP, Gueguen A et al: Defining disease activity in ankylosing spondylitis: is a combination of variables (Bath Ankylosing Spondylitis Disease Activity Index) an appropriate instrument? Rheumatology 38: 878-882, 1999.

9. Calin A, Garrett S, Whitelock H et al: A new approach to defining functional ability in ankylosing spondylitis: the development of the Bath Ankylosing Spondylitis Functional Index. J Rheumatol 21: 2281-5, 1994. 
10. van der Heijde D, Bellamy N, Calin A et al: Preliminary core sets for endpoints in ankylosing spondylitis. Assessments in Ankylosing Spondylitis Working Group. J Rheumatol 24: 2225-9, 1997.

11. van der Heijde D, Bellamy N, Calin A et al: Selection of instruments in the core set for DC-ART, SMARD, physical therapy, and clinical record keeping in ankylosing spondylitis. Progress report of the ASAS Working Group. Assessments in Ankylosing Spondylitis. J Rheumatol 26: 951-4, 1999.

12. Calin A, Mackay K, Santos H, Brophy S: A new dimension to outcome: application of the Bath Ankylosing Spondylitis Radiology Index. J Rheumatol. 26: 988-92, 1999.

13. Jones SD, Steiner A, Garrett SL, Calin A. The Bath Ankylosing Spondylitis Patient Global Score (BAS-G): Br J Rheumatol. 35: 66-71, 1996.

14. Jenkinson TR, Mallorie PA, Whitelock $\mathrm{H}$ et al: Defining spinal mobility in ankylosing spondylitis (AS). The Bath AS Metrology Index. J Rheumatol 21: 1694-8, 1994.

15. Doward LC, Spoorenberg A, Cook SA et al: Development of the ASQoL: a quality of life instrument specific to ankylosing spondylitis. Ann Rheum Dis 62: 20-6, 2003.

16. Daltroy LH, Larson MG, Roberts WN, Liang $\mathrm{MH}$ : A Modification of the Health Assessment Questionnaire for the Spondyloarthropathies. J Rheumatol 17: 946-50, 1990.

17. Guillemin F, Challier B, Urlacher F et al: Quality of life in ankylosing spondylitis: validation of the ankylosing spondylitis Arthritis Impact Measurement Scales 2, a modified Arthritis Impact Measurement Scales Questionnaire. Arthritis Care Res 12: 157-62, 1999.

18. Dougados M, Gueguen A, Nakache JP et al: Evaluation of a functional index and an articular index in ankylosing spondylitis. J Rheumatol 15: 302-7, 1998.

19. Haywood KL, Garratt AM, Dziedzic K, Dawes PT: Patient centered assessment of ankylosing spondylitis-specific health related quality of life: evaluation of the Patient Generated Index. J Rheumatol 30: 764-73, 2003.

20. Haywood KL, M Garratt A, Jordan K et al: Disease-specific, patient-assessed measures of health outcome in ankylosing spondylitis: reliability, validity and responsiveness. Rheumatology (Oxford) 41: 1295-302, 2002

21. Averns HL, Oxtoby J, Taylor HG, Jones PW, Dziedzic K, Dawes PT: Radiological outcome in ankylosing spondylitis: use of the Stoke Ankylosing Spondylitis Spine Score (SASSS). Br J Rheumatol 35: 373-6, 1996.

22. Creemers MC, Franssen MJ, van 't Hof MA, Gribnau FW, van de Putte LB, van Riel PL: A radiographic scoring system and identification of variables measuring structural damage in ankylosing spondylitis [thesis]. Nijmegen (The Netherlands): University of Nijmegen;1993.

23. Wanders AJ, Landewe RB, Spoorenberg A, Dougados M, van der Linden S, Mielants H, et al: What is the most appropriate radiologic scoring method for ankylosing spondylitis? A comparison of the available methods based on the Outcome Measures in Rheumatology Clinical Trials filter. Arthritis Rheum 50: 2622-32, 2004

24. Ciconelli RM, Ferraz MB, Santos W et al: Tradução para a língua portuguesa e validação do questionário genérico de avaliação de qualidade de vida SF-36 (Brasil SF-36). Rev. Bras Reumatol 39:143-50, 1999.

25. Haywood KL, Garratt AM, Dziedzic K, Dawes PT: Generic measures of health-related quality of life in ankylosing spondylitis: reliability, validity and responsiveness. Rheumatology (Oxford) 41: 1380-7, 2002

26. van Tubergen A, Landewe R, Heuft-Dorenbosch L et al: Assessment of disability with the World Health Organisation Disability Assessment Schedule II in patients with ankylosing spondylitis. Ann Rheum Dis 62 :140-5, 2003.

27. OMERACT IV Conference. Cancun, Mexico. J Rheumatol Suppl, 1998.

28. van der Heijde D, Braun J, McGonagle D, Siegel J: Treatment trials in ankylosing spondylitis: current and future considerations. Ann Rheum Dis 61 (Suppl III): iii24-iii32, 2002.

29. Ruof J, Stucki G: Comparison of the Dougados Functional Index and the Bath Ankylosing Spondylitis Functional Index. A literature review. J Rheumatol 26 : 955-60, 1999.

30. Mander M, Simpson JM, McLellan A et al: Studies with an enthesis index as a method of clinical assessment in ankylosing spondylitis.Ann Rheum Dis 46: 197-202, 1987.

31. Heuft-Dorenbosch L, Spoorenberg A, van Tubergen A et al: Assessment of enthesitis in ankylosing spondylitis. Ann Rheum Dis $62: 127-32,2003$

32. Spoorenberg A, van der Heijde D, de Klerk E et al: Relative value of erythrocyte sedimentation rate and C-reactive protein in assessment of disease activity in ankylosing spondylitis. J Rheumatol 26: 980-4, 1999.

33. Braun J, Baraliakos X, Golder W et al: Magnetic resonance imaging examinations of the spine in patients with ankylosing spondylitis, before and after successful therapy with infliximab: evaluation of a new scoring system. Arthritis Rheum 48: 11261136, 2003.

34. van Tubergen A, Coenen J, Landewe R, Spoorenberg A, Chorus A, Boonen A, van der Linden S, van der Heijde D: Assessment of fatigue in patients with ankylosing spondylitis: a psychometric analysis. Arthritis Rheum 47: 8-16, 2002.

35. Anderson JJ, Baron G, van der Heijde D, Felson DT, Dougados $\mathrm{M}$ : Ankylosing spondylitis assessment group preliminary definition of short-term improvement in ankylosing spondylitis. Arthritis Rheum 44: 1876-86, 2001.

36. Braun J, Pham T, Sieper J, Davis J, van der Linden S, Dougados $\mathrm{M}$, van der Heijde D; ASAS Working Group. International ASAS consensus statement for the use of anti-tumour necrosis factor agents in patients with ankylosing spondylitis. Ann Rheum Dis 62: 817-24, 2003. 